\title{
Faixa de suficiência e definição da posição da folha na avaliação do estado nutricional de pimenta-longa
}

\section{Sufficiency range and foliar position for evaluation of long pepper nutritional status}

\author{
Paulo Guilherme Salvador WADT'1 ${ }^{1}$ Lucielio Manoel da SILVA ${ }^{2}$
}

${ }^{1}$ Autor para correspondência. Eng. Agrônomo, Doutor em Solos e Nutrição de Plantas, Centro de Pesquisa Agroflorestal de Rondônia - Embrapa Rondônia, Rodovia BR-364, km 5,5, Zona Rural, Caixa Postal: 127 CEP: 76815-800 - Porto Velho - RO. E-mail: paulo.wadt@embrapa.br.

${ }^{2}$ Eng. Agrônomo, Mestrado em Genética Vegetal, Embrapa Acre, Rio Branco - AC. E-mail: lucielio.silva@embrapa.br

\section{Recebido em: 27-03-2015; Aceito em: 27-03-2016}

\section{Resumo}

O objetivo deste trabalho foi determinar a faixa de suficiência e definir a folha para avaliação do estado nutricional da pimenta-longa. Inicialmente, foram amostradas folhas de 163 acessos de pimenta-longa do banco de germoplasma da Embrapa. Em cada amostra, foram determinados teores dos nutrientes foliares e depois obtiveram-se as normas CND. A seguir, determinaram-se pelo método CND subconjuntos de plantas nutricionalmente equilibradas para um dado nutriente, do qual se obteve o intervalo de confiança a 95\%, considerado este a faixa de suficiência, as quais foram 41,5 a 42,6; 2,3 a 2,4; 14,7 a 15,6; 10,7 a 11,3 e 2,7 a

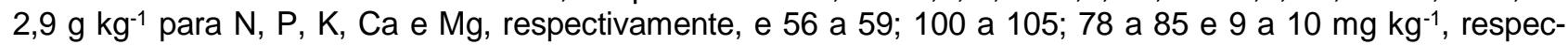
tivamente, para $\mathrm{Zn}, \mathrm{Fe}, \mathrm{Mn}$ e $\mathrm{Cu}$. No segundo momento, plantas de vinte parcelas experimentais, que receberam a mesma adubação anual, tiveram as folhas totalmente expandidas amostradas, do ápice para a base do galho: P1- primeira folha; P2 - segunda folha; P3- terceira folha e P4, quarta folha. Foram comparadas as médias e a variabilidade entre as diferentes posições, com base nos teores foliares e índices CND multivariados. Para a maioria dos nutrientes avaliados, não houve efeito da posição de amostragem, sendo que, na posição P1, houve maior concordância entre o estado nutricional determinado pelo CND e pelo método convencional, além de menor variabilidade amostral, sendo esta a posição de amostragem indicada.

Palavras-chave adicionais: CND; diagnose foliar; Piper hispidinervium.

\begin{abstract}
The objective of this work was to determine the sufficiency range and the best foliar sample position for nutritional status evaluation of long pepper. Initially, foliar samples of 163 genetic materials cultivated in germplasm collection of Embrapa were taken for nutrient content analysis and to generate CND norms. After, we obtained subgroups of balanced nutritionally for each nutrient. Following, we calculated a confidence interval at $95 \%$ for median content of nutrient, which was considered the sufficiency range: 41,5 42,6, 2,3 - 2,4, 14,7 - 15,6, 10,7 - 11,3 and 2,7 - 2,9 $\mathrm{g} \mathrm{kg}^{-1}$ for $\mathrm{N}, \mathrm{P}, \mathrm{K}, \mathrm{Ca}$ and $\mathrm{Mg}$ respectively and 56 - 59 , 100 - 105, 78- 85 and 9 - 10 mg kg-1, respectively for $\mathrm{Zn}, \mathrm{Fe}, \mathrm{Mn}$ e $\mathrm{Cu}$. In a second moment, twenty plots of a trial who received the same fertilized were sampled for positioning, from the apex to the base of the branch: P1- first leaf fully expanded, P2 - second leaf fully expanded, P3- third leaf fully expanded and P4 - fourth leaf fully expanded. We compared median and variability of nutrient content and CND indices between different leaf positions. No effect of leaf position was observed for most nutrients, with the P1 position was greater agreement for nutritional status determining for sufficiency range and CND indices and lower sample variability also. The first leaf fully expanded was indicated for nutritional evaluation of long pepper.
\end{abstract}

Additional keywords: CND; foliar diagnosis; Piper hispidinervium.

\section{Introdução}

O gênero Piper, família Piperaceae, contém mais de 1.000 espécies que se distribuem no subbosque e na vegetação secundária de florestas tropicais, principalmente na Ásia e na América Tropical, sendo suas plantas utilizadas para diferentes propósitos, como alimento, ornamental, perfumaria, venenos naturais para peixes e insetos, alucinógenos e propósitos medicinais. Muitas espécies tam- bém apresentam compostos secundários, como fenilpropenoides voláteis, como o safrol e o dilapiol (Wadt et al., 2004).

Na Amazônia sul-ocidental, o gênero Piper é particularmente abundante, sendo conhecida mais de 76 espécies, dentre as quais a "pimenta-longa" ( $P$. hispidinervium), espécie identificada na década de 1970 por pesquisadores do Instituto Nacional de Pesquisas da Amazônia (INPA) e que ocorre naturalmente como espécie invasora em áreas cultiva- 
das ou abandonadas na bacia do Acre.

O principal produto comercial do cultivo de pimenta-longa é o safrol, cuja produção depende da produtividade da biomassa, do rendimento de óleo essencial por unidade de biomassa e do teor de safrol no óleo essencial.

Em condições naturais, a variabilidade do teor de safrol no óleo essencial tem sido constatada ser baixa, com coeficiente de variação de 2,58\% (Miranda, 2001). No outro extremo, a produção de biomassa tem variado sob diferentes condições experimentais de $799 \mathrm{~kg} \mathrm{ha}^{-1}$ (Silva et al., 2001) a $7.153 \mathrm{~kg} \mathrm{ha}^{-1}$ (Figueiredo et al., 2001), sendo, provavelmente, a disponibilidade de nutriente um dos fatores determinantes desta variabilidade, embora estudos conduzidos até 0 momento não sejam conclusivos.

Sousa et al. (2001) relataram resposta da pimenta-longa à aplicação de fósforo, de potássio apenas na presença de calagem, e de nitrogênio na ausência de calagem. Por sua vez, Wadt \& Pacheco (2006) destacam que a adubação nitrogenada não proporcionou maior produtividade de biomassa em cultivos experimentais de pimenta-longa em diferentes espaçamentos.

Por outro lado, Wadt (2008) sugeriu que a análise foliar deveria ser mais amplamente utilizada em programas de recomendação de adubação, embora destaque que ensaios de calibração convencionais sejam onerosos e de difícil condução quando se pretende determinar os valores críticos para adoção do método do nível crítico ou das faixas de suficiência na interpretação do estado nutricional, dada a necessidade de repetidos ensaios em vários anos e locais.

Alternativas ao método do nível crítico tem sido o Sistema Integrado de Diagnose e Recomendação (DRIS), porém exigindo sistemas computacionais mais complexos para sua utilização (Mourão Filho, 2004). Alternativa para a obtenção dos valores críticos ou das faixas de suficiência tem sido o método da Chance Matemática, que aplica distribuições de probabilidades a dados obtidos de lavouras comerciais (Wadt et al., 1998; Kurihara, 2004; Urano et al., 2007); entretanto, necessitando do acompanhamento da produtividade das lavouras monitoradas.

O próprio sistema DRIS, com uso de relações bivariadas, tem sido utilizado como ferramenta para a obtenção dos valores críticos dos nutrientes (Guindani et al, 2007), com relativo sucesso. No mesmo sentido, o método da Diagnose da Composição Nutricional (CND) (Parent \& Dafir, 1992) tem sido utilizado para a obtenção de valores de referência, método também denominado de DRIS para relações multivariadas.

Para a pimenta-longa, por se tratar de uma espécie ainda em processo de domesticação, os estudos são ainda mais escassos, havendo poucas informações sobre a nutrição desta espécie, com resultados ainda incongruentes (Sousa et al., 2001; Viegas et al., 2004; Wadt \& Pacheco, 2006).

Neste sentido, o objetivo deste trabalho foi obter as faixas de suficiência de nutrientes e determinar a posição de amostragem das folhas para subsidiar estudos de nutrição mineral e a recomendação de adubação.

\section{Material e métodos}

Para a geração das normas CND, foram amostrados 163 acessos de plantas sadias de pimenta-longa do banco de germoplasma da Embrapa Acre, com quatro anos de idade, no período de novembro a dezembro de 2010. As populações naturais e também as cultivadas com pimenta-longa apresentam inflorescências durante todo o ano, em diferentes estádios de desenvolvimento; contudo, no período de início da maior precipitação pluvial, é comum observar maior crescimento da biomassa aérea, com expansão foliar. Assim, não se pode atribuir um estádio fenológico único à cultura, sendo que a amostragem foi realizada no início do maior ciclo de crescimento vegetativo da parte aérea.

Estes acessos foram originados de coletas de material vegetal realizadas durante os anos de 1990 a 2005, em áreas agrícolas e pastagens, predominantemente em seu centro de origem, no Vale do Rio Acre, no sudoeste da Amazônia.

As plantas amostradas foram cultivadas em Argissolo Vermelho-Amarelo e receberam anualmente $72 \mathrm{~kg} \mathrm{ha}^{-1}$ de $\mathrm{N}, 45 \mathrm{~kg} \mathrm{ha}^{-1}$ de $\mathrm{P}_{2} \mathrm{O}_{5}$ e $100 \mathrm{~kg} \mathrm{ha}^{-1}$ de $\mathrm{K}_{2} \mathrm{O}$, respectivamente, nas fontes de ureia, superfosfato triplo e cloreto de potássio.

De cada acesso, foram coletadas aproximadamente 20 plantas, e em cada planta, foram tomadas 20 folhas no terço mediano das plantas, em todas as posições cardiais, sempre se coletando a primeira folha totalmente expandida (Posição P1).

Após a coleta, as amostras foliares foram secas em estufa com circulação forçada até massa constante, a $65^{\circ} \mathrm{C}$ e depois de secas, trituradas em moinho. Nas amostras, foram analisados os teores de cálcio $(\mathrm{Ca})$, magnésio $(\mathrm{Mg})$, manganês $(\mathrm{Mn})$, ferro $(\mathrm{Fe})$, zinco $(\mathrm{Zn})$ e cobre $(\mathrm{Cu})$ por espectrofotometria de absorção atômica, potássio $(K)$ por fotometria de chama e fósforo $(P)$ por espectrofotometria de luz visível, após extração em solução nitroperclórica. O nitrogênio $(N)$ total foi determinado, após digestão sulfúrica, pelo método de Kjehdahl (Carmo et al., 2000).

Após a determinação dos teores foliares, estes foram ajustados para a mesma unidade $\left(\right.$ dag $\mathrm{kg}^{-1}$ ) e, a seguir, calculou-se o valor R, dado pela diferença entre 100 e o somatório dos teores dos nutrientes nas folhas $(R=100-(v N+v P+v K+$ $+\mathrm{vCa}+\mathrm{vMg}+\mathrm{vZn}+\mathrm{vFe}+\mathrm{vMn}+\mathrm{vCu})$ ), sendo que $R$ representa o conteúdo de massa seca da amostra e de outros nutrientes não avaliados e, vN, vP, vK, $\mathrm{vCa}, \mathrm{vMg}, \mathrm{vZn}, \mathrm{vFe}, \mathrm{vMn}$ e vCu, respectivamente, os teores de N, P, K, Ca, Mg, Zn, Fe, Mn e Cu, todos expressos em dag $\mathrm{kg}^{-1}$.

A seguir, para cada um dos 163 acessos de pimenta-longa, foi calculada a média geométrica dos 
teores nutricionais pela expressão: $\mathrm{mGeo}=(\mathrm{vN} \times \mathrm{vP} \times$ $\times \mathrm{vK} \times \mathrm{vCa} \times \mathrm{vMg} \times \mathrm{vZn} \times \mathrm{vFe} \times \mathrm{vMn} \times \mathrm{vCu} \times \mathrm{R})^{(1 / 10)}$.

Finalmente, para cada nutriente, foi obtida sua relação multivariada, a qual consistiu no logaritmo neperiano do teor de cada nutriente pela média geométrica da amostra foliar (Parent \& Dafir, 1992). Ou seja, foi obtido o valor da relação multivariada de cada nutriente pela expressão: $\mathrm{zX}=\mathrm{LN}$ (vX/mGeo), sendo que $\mathrm{Zx}$ representa 0 valor da relação multivariada para cada um dos nutrientes avaliados ( $\mathrm{VX}$ ), expressos em dag $\mathrm{kg}^{-1}$, e correspondendo a vN, vP, vK, vCa, vMg, vZn, vFe, vMn e vCu, respectivamente, para $\mathrm{N}, \mathrm{P}, \mathrm{K}, \mathrm{Ca}, \mathrm{Mg}$, $\mathrm{Zn}, \mathrm{Fe}, \mathrm{Mn}$ e $\mathrm{Cu}$, e que são representadas por $\mathrm{zN}$, zP, zK, zCa, zMg, zZn, zFe, zMn e zCu.

As normas CND foram então obtidas deste conjunto de dados, calculando-se a média aritmética, o desvio-padrão e o número de relações multivariadas para o conjunto das relações multivariadas de cada um dos nutrientes avaliados.

A determinação do valor do índice CND multivariado foi calculado para cada nutriente e para cada amostra foliar, pela expressão (Parent \& Dafir, 1992): I_X $=(\mathrm{mX}-\mathrm{mx}) / \mathrm{dx}$, sendo que $\mathrm{mX}$ representa a relação multivariada para cada um dos nutrientes avaliados e $\mathrm{mx}$ e $\mathrm{dx}$, respectivamente, a média aritmética e o desvio-padrão da respectiva relação multivariada (normas CND multivariadas).

$O$ índice de balanço nutricional médio (IBNm) foi obtido pela média aritmética do módulo de todos os índices CND multivariados dos nutrientes $\mathrm{N}, \mathrm{P}, \mathrm{K}, \mathrm{Ca}, \mathrm{Mg}, \mathrm{Zn}, \mathrm{Fe}, \mathrm{Mn}$ e $\mathrm{Cu}$.

Para a interpretação dos índices CND, utilizou-se o critério do Potencial de Resposta à Adubação (Wadt, 2005), com duas abordagens:

a) com cinco classes para o estado nutricional:

i) equilíbrio nutricional $=\left|\mathrm{I} \_\mathrm{X}\right|<\mathrm{IBNm}$. Atribuiu-se o valor zero para esta condição;

ii) alta probabilidade de insuficiência $=\left|\mathrm{I} \_\mathrm{X}\right|>$ IBN, I_X $<0$ e I_X igual ao menor índice CND na amostra. Atribui-se o valor -2 para esta condição;

iii) baixa probabilidade de insuficiência $=|I \mathrm{X}|>$ $>$ IBN, I X $<0$ e I X maior que o menor índice CND na amostra. Atribui-se o valor -1 para esta condição;

iv) alta probabilidade de excesso $=\left|I \_X\right|>\mid B N m$, I_X > 0 e I_X igual ao maior índice CN̄D na amostra. Atribui-se o valor 2 para esta condição; e

v) baixa probabilidade de excesso $=\left|I \_X\right|>\mid B N m$, I_X > 0 e I_X menor que o maior índice CND na amostra. Atribui-se o valor 1 para esta condição.

b) com simplificação para duas classes do estado nutricional:

i) equilíbrio nutricional $=\left|I \_X\right|>\mid B N m$ e I $X>0$ ou $\left|I \_X\right|<\mid B N m$. Atribuiu-se o valor zero para esta condição; e

ii) insuficiência $=\left|I \_X\right|>\mid B N$ e I_X $<0$. Atribui-se 0 valor -1 para esta condição.

$\mathrm{Na}$ definição das faixas de suficiência do estado nutricional de pimenta-longa, para cada nutriente, foi identificado, entre todos os 163 acessos avaliados, o conjunto de plantas nutricionalmente equilibrado para cada nutriente individualmente (pela abordagem com cinco classes de interpretação). A seguir, também para cada nutriente, determinou-se o intervalo de confiança a 95\% de probabilidade, definindo-se os limites inferior e superior do respectivo intervalo de confiança como o valor mínimo e máximo da faixa do nutriente considerado como adequado, cujos valores foram expressos em $\mathrm{g} \mathrm{kg}^{-1}$ para N, P, K, Ca e Mg e em mg $\mathrm{kg}^{-1}$ para $\mathrm{Zn}, \mathrm{Fe}, \mathrm{Mn}$ e $\mathrm{Cu}$. Consequentemente, teores nutricionais abaixo do limite inferior da faixa de suficiência foram considerados como deficiência, e teores acima do limite superior da faixa de suficiência foram considerados como excesso.

Para a definição da melhor posição da folha para a avaliação do estado nutricional de pimentalonga, foram obtidas amostras compostas de folhas de pimenta-longa, de vinte parcelas experimentais, em quatro posições no galho (do ápice para a base do ramo), a saber: $\mathrm{P} 1$, primeira folha totalmente expandida; P2, segunda folha totalmente expandida; P3, terceira folha totalmente expandida e P4, quarta folha totalmente expandida.

Cada amostra composta foi constituída de aproximadamente 20 folhas coletadas na parte mediana de plantas de cada parcela, cultivadas em espaçamento de $1 \mathrm{~m} \times 1 \mathrm{~m}$, em Argissolo VermelhoAmarelo, no Câmpus experimental da Embrapa Acre, Rio Branco-AC. Estas amostras foram submetidas aos mesmos procedimentos analíticos descritos acima para as demais amostras foliares.

As médias dos teores foliares e dos índices CND de cada nutriente, em relação às quatro posições de amostragem, foram comparadas pelo teste de análise de variância (teste $\mathrm{F}$ ). Aquelas que mostram haver diferenças entre as médias, pelo teste $\mathrm{F}$ a $5 \%$, foram também avaliadas pelo teste de Tukey, a 5\%.

Avaliou-se, ainda, o número de diagnósticos concordantes quanto ao estado nutricional informado pelos métodos CND e das faixas de suficiência. Para esta avaliação, contabilizou-se o número de diagnósticos coincidentes, considerando-se coincidência quando houve:

a) diagnóstico de insuficiência pelo método CND (pela abordagem utilizando duas classes de interpretação) e de deficiência pelo método das faixas de suficiência;

b) diagnóstico de equilíbrio pelo método CND (pela abordagem utilizando duas classes de interpretação) e de adequado ou de suficiência pelo método das faixas de suficiência.

Quaisquer outras combinações de diagnósticos foram consideradas como não coincidentes. 


\section{Resultados e discussão}

A adoção de relações multivariadas simplifica a obtenção de padrões nutricionais (Tabela 1), já que, para cada nutriente, uma única norma faz-se suficiente, enquanto para relações bivariadas, considerando-se as funções na forma direta e inversa, o número de normas DRIS de cada nutriente aumenta com o número de nutrientes avaliados (n), com base na expressão " $2 \times(n-1)$ ". Isto possibilita que os cálculos para a obtenção dos índices CND multivariados se tornem simplificados e facilmente obtidos em planilhas eletrônicas.

A desvantagem das normas CND multivariadas está no fato de que seu valor tomado isoladamente tem pouca informação, o que não ocorre com as normas DRIS bivariadas, já que cada norma representa o valor ótimo e a respectiva variabilidade do quociente entre os teores de dois nutrientes.

As normas CND multivariadas permitem identificar rapidamente aqueles macronutrientes com maiores teores médios ( $\mathrm{N}, \mathrm{K}$ e $\mathrm{Ca}$ ), que, por estarem acima da média geométrica, apresentam valores positivos (Tabela 1). Para os micronutrientes, contudo, por serem expressos em dag $\mathrm{kg}^{-1}$, terão sempre valores negativos (abaixo da média geométrica), o que se torna pouco informativo quanto à demanda relativa destes nutrientes.

Os teores médios, mínimo e máximo dos nutrientes na população avaliada confirmam ser $\mathrm{N}$, $\mathrm{K}$ e $\mathrm{Ca}$ aqueles requeridos em maior concentração nos tecidos foliares (Tabela 2 ), sendo o $\mathrm{K}$ e o $\mathrm{Ca}$ os macronutrientes com maiores coeficientes de variação para os teores foliares, como também com a maior amplitude. Com relação aos micronutrientes, $\mathrm{Mn}$ e $\mathrm{Cu}$ foram os que apresentaram maior coeficiente de variação.
Tabela 1 - Normas CND multivariadas para N, P, K, Ca, $\mathrm{Mg}, \mathrm{Zn}, \mathrm{Fe}, \mathrm{Mn}$ e $\mathrm{Cu}$, e valor R, em pimenta-longa.

\begin{tabular}{lrc}
\hline Nutriente & Média & Desvio-padrão \\
\hline $\mathrm{N}$ & 2,3249 & 0,1001 \\
$\mathrm{P}$ & $-0,5716$ & 0,1333 \\
$\mathrm{~K}$ & 1,2604 & 0,2841 \\
$\mathrm{Ca}$ & 0,9703 & 0,1794 \\
$\mathrm{Mg}$ & $-0,4150$ & 0,1786 \\
$\mathrm{Zn}$ & $-1,9879$ & 0,2034 \\
$\mathrm{Fe}$ & $-1,4108$ & 0,1394 \\
$\mathrm{Mn}$ & $-1,5981$ & 0,3412 \\
$\mathrm{Cu}$ & $-3,9866$ & 0,4561 \\
\hline $\mathrm{R}$ & 5,4143 & 0,0921 \\
\hline
\end{tabular}

Para os macronutrientes, estes valores diferem daqueles indicados por Viegas et al. (2004) para plantas sadias e deficientes. O teor mínimo de $\mathrm{N}\left(33 \mathrm{~g} \mathrm{~kg}^{-1}\right)$ foi superior ao limite superior dos teores foliares para plantas consideradas sem deficiência de $\mathrm{N}$ como sendo de 19,5 $\mathrm{g} \mathrm{kg}^{-1}$ (Viegas et al., 2004).

Para $\mathrm{P}$, o valor máximo encontrado nas plantas avaliadas $\left(2,9 \mathrm{~g} \mathrm{~kg}^{-1}\right)$ foi próximo ao limite superior para plantas deficientes, considerado como sendo de 2,9 $\mathrm{g} \mathrm{kg}^{-1}$ (Viegas et al., 2004).

Há dois motivos para estas diferenças: a primeira trata da restrição de variabilidade de os materiais avaliados serem oriundos de banco de germoplasma, com as condições edáficas sendo relativamente uniformes; a segunda, trata-se das condições impostas às plantas no estudo de Viegas et al (2004), no qual a omissão dos nutrientes pode ter criado condições extremas quanto à nutrição das plantas, o que não ocorreu no presente trabalho, onde as plantas apresentavam aspecto vegetativo sadio, com ausência de estresse nutricional ou ambiental.

Tabela 2 - Teores médios, desvio-padrão, coeficiente de variação (CV) e teores mínimo e máximo para os nutrientes $\mathrm{N}, \mathrm{P}, \mathrm{K}, \mathrm{Ca}, \mathrm{Mg}$, em $\mathrm{g} \mathrm{kg}^{-1}$, e de Zn, Fe, Mn e Cu, em mg kg-1.

\begin{tabular}{lcccccc}
\hline Nutriente & Média & Mínimo & Máximo & Amplitude & Desvio-Padrão & CV \\
\hline $\mathrm{N}$ & 42,2 & 33,0 & 51,0 & 17,0 & 0,3 & 7,1 \\
$\mathrm{P}$ & 2,3 & 1,7 & 2,9 & 1,2 & 0,3 & 13,0 \\
$\mathrm{~K}$ & 15,0 & 5,0 & 21,6 & 0,0 & 3,7 & 24,6 \\
$\mathrm{Ca}$ & 11,1 & 4,9 & 17,0 & 0,0 & 2,3 & 20,7 \\
$\mathrm{Mg}$ & 2,8 & 1,5 & 3,9 & 0,0 & 0,6 & 21,4 \\
$\mathrm{Zn}$ & 58 & 35 & 93 & 0 & 13 & 22,4 \\
$\mathrm{Fe}$ & 102 & 63 & 173 & 0 & 16 & 15,7 \\
$\mathrm{Mn}$ & 89 & 46 & 218 & 0 & 37 & 41,6 \\
$\mathrm{Cu}$ & 9 & 3 & 16 & 0 & 3 & 33,3 \\
\hline
\end{tabular}

Neste sentido, consideradas as condições extremas dos ensaios de omissão de nutrientes, a adoção dos valores obtidos de plantas sadias e nutricionalmente equilibradas para a interpretação do estado nutricional de plantas de pimenta-longa pode ser o mais indicado (Tabela 3), e mesmo que sendo distintos daqueles publicados por Viegas et al. (2004), representam condições normais esperadas para o desenvolvimento de lavouras comerciais. 
Tabela 3 - Faixa de suficiência para os nutrientes $\mathrm{N}, \mathrm{P}, \mathrm{K}, \mathrm{Ca}, \mathrm{Mg}$, em g kg-1, e de $\mathrm{Zn}, \mathrm{Fe}, \mathrm{Mn}$ e Cu, em mg kg-1, obtidos neste trabalho, com base nas lavouras consideradas nutricionalmente equilibradas, e publicados na literatura, para plantas sem e com deficiência reconhecida.

\begin{tabular}{|c|c|c|c|}
\hline Nutriente & $\begin{array}{c}\text { Plantas nutricionalmente } \\
\text { equilibradas }\end{array}$ & $\begin{array}{l}\text { Viegas et al. (2004) } \\
\text { (Sem deficiência) }\end{array}$ & $\begin{array}{l}\text { Viegas et al. (2004) } \\
\text { (Deficientes) }\end{array}$ \\
\hline $\mathrm{N}$ & 41,5 a 42,6 & 16,7 a 19,5 & 8,5 a 9,5 \\
\hline$P$ & 2,3 a 2,4 & 6,4 a 7,7 & 2,3 a 2,8 \\
\hline K & 14,7 a 15,6 & 20,0 a 25,5 & 8,0 a 9,4 \\
\hline $\mathrm{Ca}$ & 10,7 a 11,3 & 14,9 a 16,7 & 9,3 a 10,6 \\
\hline Mg & 2,7 a 2,9 & 7,5 a 9,0 & 1,7 a 2,0 \\
\hline $\mathrm{Zn}$ & 56 a 59 & - . - & - - - - \\
\hline $\mathrm{Fe}$ & 100 a 105 & $-\ldots$ & $-\ldots$ \\
\hline $\mathrm{Mn}$ & 78 a 85 & $-\ldots$ & $-\ldots$ \\
\hline $\mathrm{Cu}$ & 9 a 10 & $-\cdots$ & - - - - \\
\hline
\end{tabular}

Os teores foliares e os respectivos índices CND não foram afetados pela posição da folha para os nutrientes $\mathrm{N}, \mathrm{K}, \mathrm{Mg}, \mathrm{Zn}$, Fe, $\mathrm{Mn}$ e $\mathrm{Cu}$, com base na análise de variância. As médias, desvio-padrão e respectivos coeficientes de variação indicaram que a variabilidade entre os índices CND foi superior àquela observada para os teores nutricionais (Tabela 4). Por outro lado, os teores foliares de $\mathrm{P}$ e Ca diferiram entre as posições da folha no galho de pimenta-longa (Teste $\mathrm{F}, \mathrm{P}<0,05)$ (Tabela 5).

Em trabalho com a nutrição da videira 'Itália', Terra et al. (2003) relacionam maior coeficiente de variação $(C V)$ à ocorrência de desequilíbrios nutricionais; o que pode ser inapropriado, dado que essa variabilidade pode refletir ser uma característica intrínseca da distribuição de cada nutriente no órgão avaliado.

Neste trabalho, todas as vinte parcelas avaliadas representam a mesma lavoura e condições nutricionais. Além disto, observa-se que o valor do $\mathrm{CV}$ foi fortemente dependente do método de interpretação adotado (teor nutricional ou relação multivariada) (Tabelas 4 e 5 ).

Para $\mathrm{P}$, as amostras foliares $\mathrm{P} 1$ e $\mathrm{P} 2$ apresentaram maiores teores foliares e maiores índices
CND (Tabela 5). Entretanto, para Ca, o comportamento foi distinto: a posição com maior teor foliar foi a que apresentou menor índice CND, e aquele com maior índice CND apresentou menor teor foliar (Tabela 5).

Hiroce \& Terra (1984), ao compararem os teores nutricionais no limbo e nas folhas de videiras, recomendaram que órgãos com maior teor foliar devam ser utilizados para avaliar a nutrição mineral das plantas. Se aplicado este critério, qualquer posição da folha poderia ser utilizada para a maioria dos nutrientes (Tabela 4), sendo para $P$ mais indicada a amostragem na posição $\mathrm{P} 1$ e $\mathrm{P} 2$, e para $\mathrm{Ca}$ a posição P3 e P4 (Tabela 5).

No caso do $\mathrm{Ca}$, a baixa mobilidade deste nutriente entre órgãos da planta (Prado, 2008) pode ser um dos fatores relacionados a esta diferença, uma vez que a taxa de acumulação relativa do nutriente (Wadt \& Novais, 1999) pode diferir entre as posições de amostragem, fazendo com que, nas folhas mais novas (P1 e P4), a taxa relativa de acumulação de massa seca seja maior que a taxa relativa de acumulação de $\mathrm{Ca}$, resultando em efeito de diluição de Ca nestas posições.

Tabela 4 - Médias (m) e desvios-padrão (s) para os teores e índices CND de N, K e Mg, Zn, Fe, Mn e Cu e respectivos coeficientes de variação $(\mathrm{CV})$.

\begin{tabular}{lccccccr}
\hline \multirow{2}{*}{ Nutriente } & \multirow{2}{*}{ Unidades } & \multicolumn{3}{c}{ Teor foliar } & \multicolumn{3}{c}{ Índice CND } \\
\cline { 3 - 8 } & & $\mathrm{m}$ & $\mathrm{s}$ & $\mathrm{CV}(\%)$ & $\mathrm{m}$ & $\mathrm{s}$ & $\mathrm{CV}(\%)$ \\
\hline $\mathrm{N}$ & $\mathrm{g} \mathrm{kg}^{-1}$ & 39,8 & 3,5 & 8,8 & $-1,82$ & 1,22 & $-66,9$ \\
$\mathrm{~K}$ & $\mathrm{~g} \mathrm{~kg}^{-1}$ & 15,2 & 3,0 & 19,6 & $-0,33$ & 0,70 & $-210,4$ \\
$\mathrm{Mg}$ & $\mathrm{g} \mathrm{kg}^{-1}$ & 2,9 & 0,6 & 19,5 & $-0,40$ & 0,94 & $-234,1$ \\
$\mathrm{Zn}$ & $\mathrm{mg} \mathrm{kg}^{-1}$ & 72 & 6 & 7,9 & 0,59 & 0,42 & 71,7 \\
$\mathrm{Fe}$ & $\mathrm{mg} \mathrm{kg}^{-1}$ & 85 & 24 & 28,2 & $-2,34$ & 1,81 & $-77,2$ \\
$\mathrm{Mn}$ & $\mathrm{mg} \mathrm{kg}^{-1}$ & 192 & 54 & 27,9 & 1,99 & 0,78 & 39,1 \\
$\mathrm{Cu}$ & $\mathrm{mg} \mathrm{kg}^{-1}$ & 11 & 4 & 35,1 & 0,35 & 0,70 & 201,1 \\
\hline
\end{tabular}


Tabela 5 - Médias (m) e desvios-padrão (s) para os teores e índices CND de P e K, em g kg-1, e respectivos coeficientes de variação (CV), em relação a cada posição de coleta no galho.

\begin{tabular}{|c|c|c|c|c|c|c|}
\hline \multirow{2}{*}{$\begin{array}{c}\text { Posição de } \\
\text { coleta da folha }\end{array}$} & \multicolumn{3}{|c|}{ Teor foliar* } & \multicolumn{3}{|c|}{ Índice CND* } \\
\hline & $\mathrm{m}$ & $\mathrm{s}$ & $\mathrm{CV}(\%)$ & $\mathrm{m}$ & $s$ & $\mathrm{CV}(\%)$ \\
\hline & \multicolumn{6}{|c|}{${ }^{2} \cdot(1)$} \\
\hline P1 & $3,3 a$ & 0,2 & 6,6 & $1,57 a$ & 0,90 & 57,1 \\
\hline P2 & $2,7 \mathrm{~b}$ & 0,3 & 11,1 & $0,16 \mathrm{~b}$ & 1,00 & 628,2 \\
\hline P3 & $2,3 c$ & 0,1 & 6,0 & $-0,85 c$ & 0,81 & $-94,9$ \\
\hline P4 & $2,2 \mathrm{c}$ & 0,1 & 5,5 & $-1,43 c$ & 0,60 & $-42,1$ \\
\hline $\mathrm{m}$ & 2,6 & 0,5 & 18,9 & $-0,14$ & 1,41 & $-1018,6$ \\
\hline & \multicolumn{6}{|c|}{$\mathrm{Ca}$} \\
\hline P1 & $8,8 \mathrm{c}$ & 1,5 & 16,9 & $-2,07$ & $1,01 \mathrm{a}$ & 48,6 \\
\hline P2 & $10,6 b$ & 1,4 & 13,1 & $-0,84$ & $0,42 b$ & 50,5 \\
\hline P3 & $11,5 a b$ & 1,2 & 10,1 & $-0,29$ & $0,58 \mathrm{bc}$ & 198,9 \\
\hline P4 & $12,2 a$ & 1,7 & 13,9 & $-0,06$ & $0,74 c$ & 1276,9 \\
\hline $\mathrm{m}$ & 10,8 & 1,9 & 17,6 & $-0,81$ & 1,05 & $-129,4$ \\
\hline
\end{tabular}

* Médias seguidas de mesma letra, na mesma coluna, não diferem entre si, pelo teste de Tukey, a $5 \%$ de significância.

Avaliando-se o CV para as médias e os índices CND de $\mathrm{P}$ e $\mathrm{Ca}$ entre as quatro posições de coleta, a posição P1 (primeira folha completamente expandida) foi a que apresentou menor CV para os dois indicadores (teor foliar e índice CND). Isto implica que a amostragem feita nesta posição proporciona menor aleatoriedade no teor nutricional e, portanto, maior possibilidade de refletir adequadamente 0 estado nutricional da pimenta-longa, sendo, portanto, a mais indicada. Este critério difere substancialmente de trabalhos clássicos que sugerem a escolha do órgão com maior concentração nutricional, como sugerido para culturas da batata e do tomateiro (Gallo et al., 1965; Hiroce et al., 1972).

Para os demais nutrientes, como não houve diferença entre as médias, em princípio, qualquer posição de coleta poderia ser adotada, embora também para estes nutrientes a posição $\mathrm{P} 1$ tenha apresentado menores valores de CV para a maioria dos casos avaliados.

Comparando-se o grau de concordância entre diagnósticos nutricionais produzidos para cada nutriente, em relação às diferentes posições de amostragem, observa-se que, em geral, o grau de concordância foi baixo (menor que $80 \%$ ), à exceção de $\mathrm{Zn}$ e Mn (Tabela 6).

Esta baixa concordância foi menor inclusive que aquela encontrada em estudos de cupuaçu conduzidos em diferentes condições edafoambientais e com materiais geneticamente muito distintos (Dias, 2010), diferente do presente trabalho onde há maior uniformidade do material genético e das condições de cultivo.

Tabela 6 - Grau de concordância, em porcentagem, entre diagnósticos do estado nutricional da pimenta-longa produzidos pelos métodos da faixa de suficiência e do CND, em relação à posição de amostragem das folhas.

\begin{tabular}{lrrrrr}
\hline \multirow{2}{*}{ Nutriente } & \multicolumn{5}{c}{ Posição da folha amostrada na planta } \\
\cline { 2 - 6 } $\mathrm{N}$ & $\mathrm{P} 1$ & $\mathrm{P} 2$ & $\mathrm{P} 3$ & $\mathrm{P} 4$ & Média \\
\hline $\mathrm{P}$ & 50 & 85 & 60 & 65 & 65 \\
$\mathrm{~K}$ & 100 & 95 & 70 & 40 & 76 \\
$\mathrm{Ca}$ & 85 & 60 & 70 & 45 & 65 \\
$\mathrm{Mg}$ & 55 & 55 & 80 & 90 & 70 \\
$\mathrm{Zn}$ & 60 & 70 & 60 & 70 & 65 \\
$\mathrm{Fe}$ & 100 & 100 & 100 & 100 & 100 \\
$\mathrm{Mn}$ & 80 & 70 & 100 & 10 & 75 \\
$\mathrm{Cu}$ & 100 & 70 & 70 & 85 & 100 \\
Média & 80 & 76 & 74 & 78 \\
\hline
\end{tabular}

\section{Conclusões}

O método CND para relações multivariadas, conjugado ao critério do potencial de resposta à adubação, pode ser utilizado com alternativa para a determinação da faixa de suficiência ou níveis críticos dos nutrientes.

A primeira folha completamente expandida, do ápice da planta para a base do galho, pode ser utilizada como referência para a posição de amostragem, por apresentar valores compatíveis com folhas maduras de outras posições do ramo. 
O grau de concordância entre o estado nutricional da pimenta longa definido pelos métodos CND de relações multivariadas e o método das faixas de suficiência não mostrou ser adequado, sugerindo diferenças entres estes dois métodos de diagnóstico.

\section{Referências}

Carmo CAFS, Araújo WS, Bernardi ACC, Saldanha MFC (2000) Métodos de análise de tecidos vegetais utilizados pela Embrapa Solos. Rio de Janeiro: Embrapa Solos, 41p.

Dias JRM (2010) Sistema Integrado de Diagnose e Recomendação (DRIS) para cupuaçueiros na Amazônia Sul-Ocidental. Rio Branco: Universidade Federal do Acre. 82p. (Dissertação Mestrado).

Figueiredo FJC, Rocha Neto OG, ALVES SM, Silva ESA (2001) Frequência de corte de plantas de pimenta longa (Piper hispidinervum C.DC.) para fins de produção de biomassa, extração de óleo essencial e quantificação de safrol. In. Pimentel FA, Rocha Neto OG. Workshop de encerramento do projeto Desenvolvimento de Tecnologias para Produção de Safrol a partir da Pimenta Longa (Piper hispidinervum C.DC.), 1.:2001, Rio Branco, AC. Rio Branco: Embrapa Acre; Belém: Embrapa Amazônia Oriental/DFID. p. 57-63.

Gallo JR, Coelho FAZ, Nóbrega SA (1965) Análise de folíolos e pecíolos na diagnose da nutrição de batatinha. Bragantia, 24:385-401.

Guindanim RHP, Anghinoni I, Nachtigall GR (2009) DRIS na avaliação do estado nutricional do arroz irrigado por inundação. Revista Brasileira de Ciência do Solo, 33:109-118.

Hiroce R, Bataglia OC, Gallo JR, Campos HP (1972) Amostragem em tomateiro (Lycopersicon esculentum Mill) cultivar Santa Cruz IAC-2731, para fins de análise química foliar. Ciência e Cultura, 24:242-244.

Hiroce R, Terra NM (1984) Teores de macronutrientes em pecíolo e limpo da videira 'Niagara Rosada'. In. Congresso Brasileiro de Fruticultura, 7, 1983. Florianópolis. Anais.... Florianópolis: SBF, 4:1184-1187.

Kurihara CH (2004) Demanda de nutrientes pela soja e diagnose de seu estado nutricional. Viçosa, MG, Universidade Federal de Viçosa, 101p. (Tese de Doutorado).

Miranda EM (2001) Caracterização e avaliação produtiva de uma população nativa de pimenta longa (Piper hispidinervum C.DC.) no seringal Cachoeira, AC. In. Pimentel FA, Rocha Neto OG. Workshop de encerramento do projeto Desenvolvimento de Tecnologias para Produção de Safrol a partir da Pimenta Longa (Piper hispidinervum C.DC.), 1.:2001, Rio Branco, AC. Rio Branco: Embrapa Acre; Belém: Embrapa Amazônia Oriental/DFID. p. 45-50.
Mourão Filho FAA (2004) DRIS: Concepts and applications on nutritional diagnosis in fruit crops. Sciencia Agricola, 61:550-560.

Parent LE, Dafir M (1992) A theorical concept of compositional nutriente diagnosis. Jounal of American Society for Horticultural Science, 117:239-242.

Prado RM (2008) Nutrição de Plantas. 1. ed. São Paulo: UNESP. 407 p.

Silva ESA, Rocha NETO OG, Figueiredo FJC (2001) Crescimento e Produção de óleo essencial de Pimenta Longa (Piper hispidinervum C.DC.) sob diferentes condições de manejo, no município de Igarapé-Açu, PA. In. Pimentel FA, Rocha Neto OG. Workshop de encerramento do projeto Desenvolvimento de Tecnologias para Produção de Safrol a partir da Pimenta Longa (Piper hispidinervum C.DC.), 1. Rio Branco, AC. Rio Branco: Embrapa Acre; Belém: Embrapa Amazônia Oriental/DFID. p. 90-95.

Sousa MMM, Ledo FJS, Pimentel FA (2001) Efeito da adubação e do calcário na produção de matéria seca e de óleo essencial de pimenta-longa. Pesquisa Agropecuária Brasileira, 36:405-409.

Terra MM, Guilherme MAS, Wagner RS, Paioli-Pires EJ, Pommer CV, Boteljo RV (2003) Avaliação do estado nutricional da videira "Itália" na região de Jales, SP, usando o sistema integrado de diagnose e recomendação. Revista Brasileira de Fruticultura, 25:309-314.

Urano EOM, Kurihara $\mathrm{CH}$, Maeda S, Vitorino ACT, Gonçalves MC, Marchetti ME (2007) Determinação de teores ótimos de nutrientes em soja pelos métodos Chance Matemática, sistema integrado de diagnose e recomendação e diagnose da composição nutricional. Revista Brasileira de Ciência do Solo, 31:63-72.

Viegas IJM, Brasil EC, Frazão DAC, Conceição HEO (2004) Pimenta longa: nutrição, calagem e adubação. Belém: Embrapa Amazônia Oriental. 4p. (Circular Técnica, 34)

Wadt LHO, Ehringhaus C, Kageyama PY (2004) Genetic diversity of "Pimenta Longa" genotypes (Piper spp., Piperaceae) of the Embrapa Acre germoplasm collection. Genetics and Molecular Biology, 27:74-82.

Wadt PGS (2005) Relationships between soil class and nutritional status of coffee crops. Revista Brasileira de Ciência do Solo, 29:227-234. 
Wadt PGS (2008) Análise foliar para recomendação de adubação em culturas agrícolas. In: Prado RM, Rozane DE, Vale DW, Correia MAR, Souza HA (Org.). Nutrição de plantas. Diagnose foliar em grandes culturas. Jaboticabal: Fundenesp, v. 1, p. 115-133.

Wadt PGS, Novais RF (1999) O monitoramento nutricional frente aos métodos diagnósticos no planejamento das adubações. In: Simpósio sobre Monitoramento Nutricional para a Recomendação de Adubação de Culturas, 1999 , Piracicaba. I Simpósio sobre Monitoramento Nutricional para a Recomendação de Adubação de Culturas. Piracicaba: Potafos. vol. 1. p. 1-18.
Wadt PGS, Novais RF, Alvarez-V VH, Fonseca S, Barros NF (1998) Valores de Referência Para Macronutrientes Em Eucalipto Obtidos Pelos Métodos Dris e Chance Matemática. Revista Brasileira de Ciência do Solo, 22:685-692.

Wadt PGS, Pacheco EP (2006) Efeito da adubação nitrogenada, em diferentes densidades de plantio, na produção de biomassa de Pimenta Longa (Piper hispidinervum C.DC.). Revista de Biologia e Ciências da Terra, v. 6, p. 334-340. 